Honam Mathematical J. 36 (2014), No. 2, pp. 435-454

http://dx.doi.org/10.5831/HMJ.2014.36.2.435

\title{
TOPOLOGICAL PROPERTIES OF GRAPHICAL ARRANGEMENTS
}

\author{
Thi A. Nguyen and SAngwook KIM*
}

\begin{abstract}
We show that for any graph $G$, the proper part of the intersection poset of the corresponding graphical arrangement $\mathcal{A}_{G}$ has the homotopy type of a wedge of spheres. Furthermore, we also indicate the number of spheres in the wedge, based on the number of spanning forests of $G$ and other graphs that are obtained from $G$.
\end{abstract}

\section{Introduction}

A hyperplane arrangement $\mathcal{A}$ is a finite set of affine hyperplanes in some vector space $V \cong K^{n}$, where $K$ is a field. Graphical arrangements are special hyperplane arrangements obtained from finite simple undirected graphs. We know that the graphical arrangement of a graph $G$ on vertex set $[n]:=\{1,2, \ldots, n\}$ is a subarrangement of the braid arrangement $B_{n}$ that is the graphical arrangement of the complete graph $K_{n}$. Graphical arrangement are studied in the connection with graph theory $[3,6]$. Many of the invariants associated with a graphical arrangement can be computed directly from the corresponding graph such as the characteristic polynomial ([9]).

One of the important invariants of a hyperplane arrangement $\mathcal{A}$ is the intersection poset $L(\mathcal{A})$, that is, the collection of all nonempty intersections of hyperplanes in $\mathcal{A}$ ordered by reverse inclusion. The number of regions and bounded regions of a real hyperplane arrangement is determined by its intersection poset [10] and the Betti numbers of

Received April 3, 2014. Accepted May 28, 2014.

2010 Mathematics Subject Classification. 05C88, 05 C89.

Key words and phrases. graphical arrangement, EL-shallablity.

This research was supported by Basic Science Research Program through the National Research Foundation of Korea(NRF) funded by the Ministry of Education, Science and Technology(NRF-2012R1A1A1013149).

${ }^{*}$ Corresponding author 
the complement of a complex hyperplane arrangement can be expressed using the intersection poset [4].

In this paper, we study the topological properties of the intersection poset of a graphical arrangement. Björner and Wach [2] show that if a bounded poset $P$ is EL-shellable, then the order complex $\Delta(\bar{P})$ has the homotopy type of a wedges of spheres, where $\bar{P}$ is the proper part of $P$ and the order complex $\Delta(\bar{P})$ is the abstract simplicial complex whose vertices are all elements of $\bar{P}$ and whose simplices are all finite chains of $\bar{P}$, including the empty chain. They also show that the number of spheres in the wedge can be obtained from decreasing maximal chains. A well-known example of an EL-shellable poset is $\bar{\Pi}_{n}$, the proper part of the partition lattice $\Pi_{n}$ of the $n$-element set ordered by refinement. The poset $\bar{\Pi}_{n}$ has the homotopy type of a wedge of spheres, and the number of spheres is computed by counting decreasing maximal chains (see $[5,11,12])$.

The main purpose of this paper is to show that the intersection poset of a graphical arrangement is EL-shellable. We also find the number of spheres in the wedge by using the number of spanning forests of $G$ and other graphs that are obtained from $G$.

The rest of the paper is organized as follows. Section 2 provides the basic notions and definitions which will be used throughout the paper. In Section 3, an edge-labeling is defined and shown to be an EL-labeling. Section 4 describes the number of spheres in the wedge using the number of decreasing chains. The number of regions of a graphical arrangement and the enumerative results for special cases will be given in the last section.

\section{Basic notions and definitions}

Let $G$ be a simple undirected graph on a vertex set $[n]=\{1,2, \ldots, n\}$ and $E(G)=\left\{e_{1}, e_{2}, \ldots, e_{m}\right\}$ denote the set of edges of $G$. We also use the notation $i j$ for an edge of $G$ with two vertices $i$ and $j$. Let $V=K^{n}$ be a vector space over a field $K$ with coordinates $x_{1}, x_{2}, \ldots, x_{n}$.

Definition 2.1. The graphical arrangement $\mathcal{A}_{G}$ in a vector space $V$ is the hyperplane arrangement

$$
\left\{x_{i}-x_{j}=0 \mid i j \in E(G)\right\} .
$$

In the intersection poset of the graphical arrangement $\mathcal{A}_{G}$, we also label each hyperplane $x_{i}-x_{j}=0$ by $i j$. There are two cases to label an intersection of two hyperplanes. If two hyperplanes are $i j$ and $k l$, where 


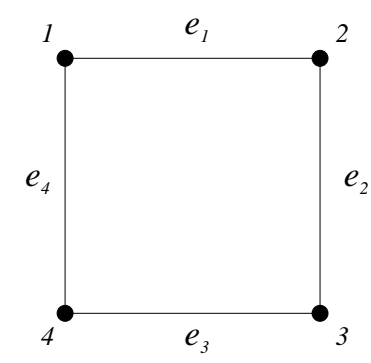

$G_{1}$

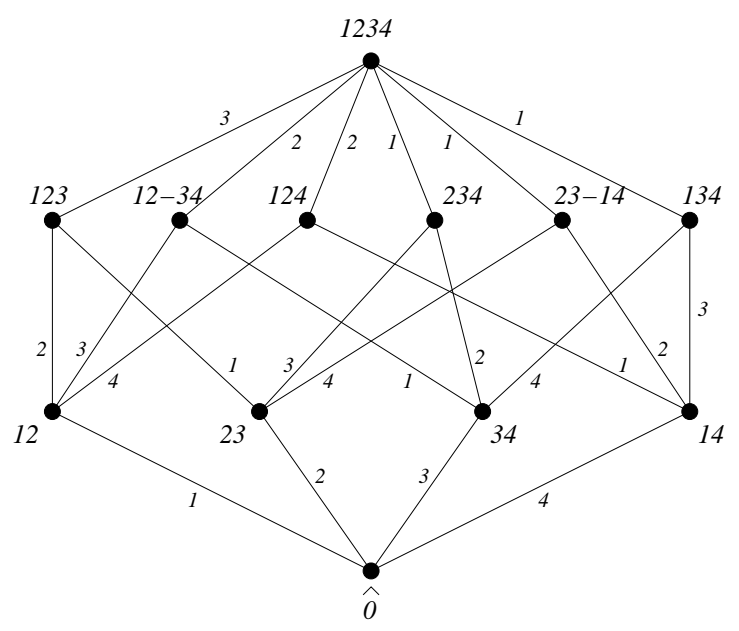

$P_{G_{1}}$

Figure 1. A graph and the intersection poset of its graphical arrangement with labeling values

$\{i, j\} \cap\{k, l\}=\emptyset$, then the common subspace is labeled by $i j-k l$. Otherwise, we may assume that $l=j$. Then the common subspace will be labeled by $i j k$. A general subspace in the intersection poset is denoted by $A=A_{1}-A_{2}-\cdots-A_{r}$, where $A_{i}, i=1,2, \ldots, r$, are the sets of indices of coordinates. We call $A_{i}$ blocks of $A$, and those indices of coordinates indices of $A$. When we get the intersection of two subspaces $H=H_{1}-H_{2}-\cdots-H_{p}$ and $K=K_{1}-K_{2}-\cdots-K_{q}$, where $H_{i}$ and $K_{i}$ are blocks of $H$ and $K$ respectively, we also have two cases to label their common subspace. It is similar to labeling common subspace of two hyperplanes. Furthermore, $K<H$ if and only if each block $K_{j}$ of $K$ is contained in some block $H_{i}$ of $H$.

Example 2.2. Consider the graph $G_{1}$ shown in Figure 1. The graphical arrangement $\mathcal{A}_{G_{1}}$ of $G_{1}$ consists of hyperplanes $x_{1}-x_{2}=0$, $x_{2}-x_{3}=0, x_{3}-x_{4}=0$, and $x_{1}-x_{4}=0$. In the intersection poset $P_{G_{1}}$ of $\mathcal{A}_{G_{1}}$, we label hyperplane $x_{1}-x_{2}=0$ by $12, x_{2}-x_{3}=0$ by $23, x_{3}-x_{4}=0$ by 34 , and $x_{1}-x_{4}=0$ by 14 . Figure 1 also shows the intersection poset $P_{G_{1}}$.

Definition 2.3. An edge-labeling of a poset $P$ is a map $\lambda: \mathcal{E}(P) \rightarrow$ $\Lambda$, where $\mathcal{E}(P)$ is the set of edges of the Hasse diagram of poset $P$ and $\Lambda$ is an ordered set. For an edge $\{s, t\} \in \mathcal{E}(P)$, we denote $\lambda(s, t)$ 
for $\lambda(\{s, t\})$. A maximal chain $m: x_{0} \lessdot x_{1} \lessdot x_{2} \lessdot \cdots \lessdot x_{t}$ of $P$ is increasing if $\lambda\left(x_{0}, x_{1}\right)<\lambda\left(x_{1}, x_{2}\right)<\cdots<\lambda\left(x_{t-1}, x_{t}\right)$. Similarly, $m$ is decreasing if $\lambda\left(x_{0}, x_{1}\right)>\lambda\left(x_{1}, x_{2}\right)>\cdots>\lambda\left(x_{t-1}, x_{t}\right)$. The maximal chain $m^{\prime}:=y_{0} \lessdot y_{1} \lessdot y_{2} \lessdot \cdots \lessdot y_{s}$ is less than $m$ in lexicographic partial order if

- $\lambda\left(x_{i}, x_{i+1}\right)=\lambda\left(y_{i}, y_{i+1}\right)$ for $i=0, \ldots, s-1$ and $s<t$, or

- $\lambda\left(x_{i}, x_{i+1}\right) \neq \lambda\left(y_{i}, y_{i+1}\right)$ for some $i$ and $\lambda\left(x_{i}, x_{i+1}\right)<\lambda\left(y_{i}, y_{i+1}\right)$ for the least such $i$.

Definition 2.4. An edge-labeling is called an EL-labeling (edge lexicographical labeling) if for every interval $[x, y]$ in poset $P$, there is a unique increasing maximal chain, which lexicographically precedes all other maximal chains of $[x, y]$.

Björner and Wachs [2] showed the following fundamental result about the EL-labeling.

Theorem 2.5. If a bounded poset $P$ admits an EL-labeling, then the order complex $\Delta(\bar{P})$ has the homotopy type of a wedge of spheres. Furthermore, the number of $i$-spheres is the number of decreasing maximal $(i+2)$-chains of $P$.

\section{The edge-labeling}

In this section, we will define an edge-labeling of the intersection poset of the graphical arrangement for graph $G$, and show that it is an EL-labeling.

Let $P_{G}$ be the intersection poset of the graphical arrangement $\mathcal{A}_{G}$ of $G$ and $H$ and $K$ be two elements in $P_{G}$ where $H$ covers $K$. We label an edge $\{K, H\}$ by

$$
\lambda(K, H)=\min \left\{i: K \cap e_{i}=H\right\} .
$$

Theorem 3.1. The labeling $\lambda$ is an EL-labeling.

Proof. Let $M$ and $N$ be two elements in the poset $P_{G}$ such that $N<M$. We construct a maximal chain

$$
\mathcal{C}=\left\{N=N_{0} \lessdot N_{1} \lessdot N_{2} \lessdot \cdots \lessdot N_{l}=M\right\}
$$

where $N_{i+1}$ is an element covering $N_{i}$ satisfying

$$
\lambda\left(N_{i}, N_{i+1}\right)=\min \left\{\lambda\left(N_{i}, N^{\prime}\right): N_{i} \lessdot N^{\prime}<M\right\} .
$$

We claim that $C$ is the only increasing maximal chain from $N$ to $M$, which lexicographically precedes all other maximal chains of $[N, M]$. 
Let $A, B$ and $C$ be three elements in $\mathcal{C}$ such that $A \lessdot B \lessdot C$. If $\lambda(A, B)=x$ and $\lambda(B, C)=y$, then $A \cap e_{x}=B$ and $B \cap e_{y}=C$. Since the hyperplane $e_{y}$ doesn't contain the subspace $A, A \cap e_{y}$ is another covering of $A$. By the choice of $B, x=\lambda(A, B)<\lambda\left(A, A \cap e_{y}\right) \leq y$. Thus $\mathcal{C}$ is increasing.

Let $\mathcal{D}=\left\{N=M_{0} \lessdot M_{1} \lessdot M_{2} \lessdot \cdots \lessdot M_{l}=M\right\}$ be another maximal chain in $[N, M]$ and let $k$ be the smallest index such that $N_{k} \neq M_{k}$. Let $\lambda\left(N_{k-1}, N_{k}\right)=z_{1}$ and $\lambda\left(M_{k-1}, M_{k}\right)=z_{2}$. If $z_{1}=z_{2}$, then

$$
N_{k}=N_{k-1} \cap e_{z_{1}}=N_{k-1} \cap e_{z_{2}}=M_{k}
$$

which is impossible. Thus $z_{1}<z_{2}$. Since $e_{z_{1}}<M$ and $e_{z_{1}} \nless M_{k}$ in $P_{G}$, we have $M_{k}<M_{t} \cap e_{z_{1}}=M_{t+1}<M$ for some $t$. But then $\lambda\left(M_{t}, M_{t+1}\right) \leq z_{1}<z_{2}=\lambda\left(M_{k-1}, M_{k}\right)$. Therefore the maximal chain $\mathcal{D}$ is not increasing.

By the construction of the maximal chain $\mathcal{C}$, it is obvious that $\mathcal{C}$ lexicographically precedes all other maximal chains of $[N, M]$.

Remark 3.2. Theorem 3.1 can be obtained from [7] since the intersection poset of a graphical arrangement is a geometric lattice. We provide an elementary proof for completeness.

Example 3.3. Figure 1 shows a graph $G_{1}$ and its intersection poset $P_{G_{1}}$ with the labeling values.

Since $P_{G}$ is the intersection poset of a hyperplane arrangement, all maximal chains in $P_{G}$ have the same length $n-c(G)$, where $c(G)$ is the number of connected components in $G$. Thus the order complex $\Delta\left(\overline{P_{G}}\right)$ has the homotopy type of a wedge of spheres of the same dimension $n-c(G)-2$.

Remark 3.4. In [6], Stanley gives a labeling rule for the graphical arrangement of a chordal graph, and the labeling values are from the set $[l]$ where $l$ is the rank of the intersection poset. In our paper, we also give a labeling rule for graphical arrangements, and the labeling values are from the set $[n]$ where $n$ is the number of hyperplanes in the arrangement. In general, the two labeling rules are different. For example, see the chordal graph $G_{2}$ in Figure 2. By the labeling rule of this paper, the unique increasing maximal chain in $[\hat{0}, \hat{1}]$ of the intersection poset is $\hat{0} \lessdot 12 \lessdot 123 \lessdot 1234$. If we choose that maximal chain for the M-chain in the labeling of Stanley, then we get $\hat{0} \lessdot 34 \lessdot 134 \lessdot 1234$ for a decreasing maximal chain while it is not decreasing in the labeling of this paper. 


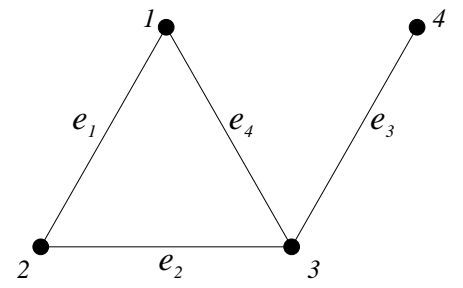

Figure 2. A chordal graph $G_{2}$

\section{The number of decreasing maximal chains}

In this section, we will find the number of decreasing maximal chains in the poset $P_{G}$.

Let $\mathcal{M}:=\hat{0} \lessdot X_{1} \lessdot X_{2} \lessdot X_{3} \lessdot \cdots \lessdot X_{l} \lessdot \hat{1}$ be a maximal chain in $[\hat{0}, \hat{1}]$. We associate $\mathcal{M}$ with the ordered string of labeling values:

$$
\lambda(\mathcal{M})=\left[\lambda\left(\hat{0}, X_{1}\right), \lambda\left(X_{1}, X_{2}\right), \ldots, \lambda\left(X_{l}, \hat{1}\right)\right] .
$$

Since $P_{G}$ is the intersection poset of a hyperplane arrangement, the labeling values $\lambda\left(\hat{0}, X_{1}\right), \lambda\left(X_{1}, X_{2}\right), \ldots, \lambda\left(X_{l}, \hat{1}\right)$ are corresponding to hyperplanes or edges of $G$. For example, we can associate the maximal chain $\mathcal{M}:=\hat{0} \lessdot 12 \lessdot 123 \lessdot 1234$ of Figure 1 with the string of edges $[12,23,34]$.

Definition 4.1. A string $X$ of $l\left(P_{G}\right)$ edges in $G$ is associated with a maximal chain if all the edges of $X$ are used to find labeling values of a maximal chain. Furthermore, $X$ is associated with a decreasing maximal chain if the labeling values corresponding to the edges are decreasing.

Definition 4.2. A spanning tree of a connected graph $G$ is a subgraph that connects all the vertices of $G$ and has no cycle. For a general graph $G$, a subgraph of $G$ formed by the union of spanning trees in all connected components is called a spanning forest of $G$.

The following lemma describes the condition when a set corresponds to a decreasing maximal chain.

Lemma 4.3. Let $A$ be a set of edges in $G$. Then we can arrange egdes in $A$ to get a string $X$ that can be associated with a decreasing maximal chain if and only if

- The edges in $A$ form a spanning forest in $G$.

- A doesn't contain $C \backslash e_{j}$, the set of all edges of a cycle $C$ except the edge $e_{j}$ with the smallest label in $C$. 
Proof. If we can arrange edges in $A$ to get a string $X$ associated with a decreasing maximal chain $\mathcal{M}$, then it is easy to see that $A$ must contain edges that can make a spanning forest in $G$.

Assume that $A$ contains $C \backslash e_{j}$ where $C$ is a cycle of $G$ and $j$ is the smallest label of edges in $C$. Let $S$ be an element of the poset $P_{G}$, and $S$ is the intersection of some hyperplanes (or edges) in $A$ such that the set of edges making $S$ contains all edges in cycle $C$ except $e_{j}$ and another edge $e_{k} \in C$. By the condition of $j$, we have $j<k$.

Let $v_{1}, v_{2}, \ldots, v_{m}$ be all vertices of $C$.

If $e_{j}$ and $e_{k}$ share one vertex $v_{t}$, then $v_{1} v_{2} \cdots \hat{v}_{t} \cdots v_{m}<S$ in $P_{G}$, where $\hat{v}_{t}$ means that we don't have $v_{t}$ in that subspace. Let $T$ be the smallest element in $\mathcal{M}$ such that $T>S$ and $v_{1} v_{2} \cdots v_{m}<T$. If $R$ is an element in $\mathcal{M}$ covered by $T$, then $R \cap e_{k}=R \cap e_{j}=T$. By the labeling rule, $\lambda(R, T) \leq j<k$. Therefore, $e_{k}$ is not used to find a labeling value. So $X$ is not associated with a maximal chain.

If $e_{j}$ and $e_{k}$ share no vertex, then $A_{1}-A_{2}<S$, where $A_{1} \cup A_{2}=C$ and $e_{j}, e_{k}$ can connect these two blocks. Similar to the previous case, $e_{k}$ can not be used to find a labeling value.

On the other side, if the edges in $A$ form a spanning forest in $G$ and $A$ doesn't contain $C \backslash e_{j}$, then we arrange edges of $A$ in decreasing order of labels to get a string $X$. We claim that $X$ can be associated with a decreasing maximal chain. Since edges in $X$ are arranged in decreasing order of labels, we only need to prove that all edges in $X$ are used to find labeling values.

Let $e_{w}$ be an edge of $X$, and $P=e_{w} \cap Q$ where $Q$ is the subspace formed by getting the intersection of hyperlanes standing before $e_{w}$ in $X$. If $e_{w}$ doesn't belong to any cycle of $G$, then it is obvious that $\lambda(Q, P)=w$. If $e_{w}$ belongs to a cycle of $G$, then $e_{w}$ is not used to find a labeling value when there is another edge $e_{z}$ such that $Q \cap e_{z}=P$ and $z<w$. That means $e_{z}, e_{w}$ and some other edges appearing before $e_{w}$ in $X$ form a cycle of $G$. Since $X$ doesn't contain a cycle, $e_{z}$ doesn't belong to $X$. If there is an edge $e_{z^{\prime}}$ appearing before $e_{w}$ in $X$ and $z^{\prime}<z<w$, we get a contradiction because all edges in $X$ are in decreasing order of labels. So there doesn't exist such edge $e_{z^{\prime}}$. Then $X$ contains $C \backslash e_{z}$ where $C$ is the cycle above and $z$ is the smallest label of edges in $C$. It is impossible. Therefore, $e_{w}$ is used to find a labeling value.

Example 4.4. For $A=\left\{e_{4}=14, e_{2}=23, e_{3}=34\right\}$ in Figure 1 , we cannot get a string that is associated with a decreasing maximal chain from $A$. 
By Lemma 4.3, the number of decreasing maximal chains of $P_{G}$ is the same as the the number of sets $A$ satisfying conditions of Lemma 4.3.

Let $\mathcal{B}_{G}$ be the set of all spanning forests in $G$. For each cycle $C$ of graph $G$. Let $x_{C}=\min \left\{j: e_{j}\right.$ is an edge of $\left.C\right\}$, and $\mathcal{B}_{x_{C}}$ be the set of spanning forests containing $C \backslash e_{x_{C}}$. Then the following theorem follows immediately.

Theorem 4.5. The number of decreasing maximal chains of the poset $P_{G}$ is

$$
\left|\mathcal{B}_{G}\right|-\left|\cup_{C: \text { cycle of } G} \mathcal{B}_{x_{C}}\right| \text {. }
$$

Furthermore, the order complex $\Delta\left(\overline{P_{G}}\right)$ has the homotopy type of a wedge of $\left|\mathcal{B}_{G}\right|-\left|\cup_{C \text { :cycle of } G} \mathcal{B}_{x_{C}}\right|$ spheres of dimension $n-c(G)-2$ where $c(G)$ is the number of connected components of $G$.

Example 4.6. Let $G_{1}$ be the graph shown in Figure 1. There are three decreasing maximal chains $\hat{0} \lessdot 34 \lessdot 234 \lessdot 1234, \hat{0} \lessdot 14 \lessdot 23-14 \lessdot 1234$ and $\hat{0} \lessdot 14 \lessdot 134 \lessdot 1234$. This coincides with the result of Theorem 4.5.

Lemma 4.7. Let $C_{1}, C_{2}, \ldots, C_{k}$ be cycles of $G$. If there is no $e_{x_{C_{i}}}$ that lies on $C_{j}$ where $i \neq j$, then

$$
\left|\mathcal{B}_{x_{C_{1}}} \cap \mathcal{B}_{x_{C_{2}}} \cap \cdots \cap \mathcal{B}_{x_{C_{k}}}\right|=\left|\mathcal{B}_{G / C_{1}, C_{2}, \ldots, C_{k}}\right|,
$$

where $G / C_{1}, C_{2}, \ldots, C_{k}$ is the graph obtained from $G$ by contracting all edges in $C_{1}, C_{2}, \ldots, C_{k}$. Otherwise, $\left|\mathcal{B}_{x_{C_{1}}} \cap \mathcal{B}_{x_{C_{2}}} \cap \cdots \cap \mathcal{B}_{x_{C_{k}}}\right|=0$.

Proof. The first statement follows from the definition of $\mathcal{B}_{x_{C}}$.

For the second statement, assume that $e_{C_{i}}$ lies on $C_{j}$ where $i \neq j$. If $e_{C_{i}} \neq e_{C_{j}}$, then it is obvious that $\left|\mathcal{B}_{x_{C_{i}}} \cap \mathcal{B}_{x_{C_{j}}}\right|=0$. If $e_{C_{i}}=e_{C_{j}}$, then any subgraph of $G$ that contains $C_{i} \backslash e_{C_{i}}$ and $C_{j} \backslash e_{C_{j}}$ always has a cycle $\left(C_{i} \cup C_{j}\right) \backslash e_{C_{i}}$ and so $\left|\mathcal{B}_{x_{C_{i}}} \cap \mathcal{B}_{x_{C_{j}}}\right|=0$. Thus the result follows.

Example 4.8. Let $G_{3}$ be the graph shown in Figure 3(a). Let $C$ be the cycle of $G_{3}$ with vertices $1,2,3,4$. See Figure 3(c) for the spanning trees of $G_{3}$ that doesn't contain $e_{x_{C}}=e_{1}$ and contains all other edges of $C$. Those spanning trees are corresponding to spanning trees of graph $G_{3} / C$ shown in Figure 3(d).

Theorem 4.5 can be rephrased in the following form using Lemma 4.7 and Principle of inclusion and exclusion.

Theorem 4.9. The number of decreasing maximal chains of the poset $P_{G}$ is

$$
\left|\mathcal{B}_{G}\right|-\sum_{j=1}^{c}(-1)^{j-1} \sum_{i_{1}, i_{2}, \ldots, i_{j}}\left|\mathcal{B}_{G / C_{i_{1}}, C_{i_{2}}, \ldots, C_{i_{j}}}\right|,
$$




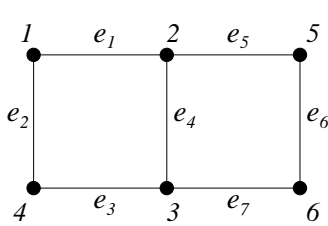

(a)

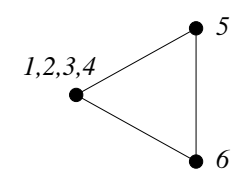

(b)

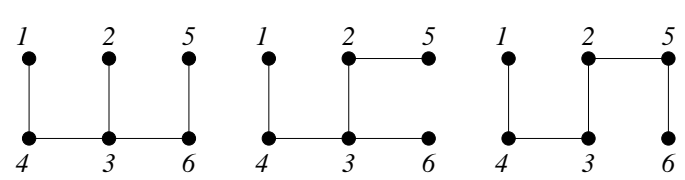

(c)
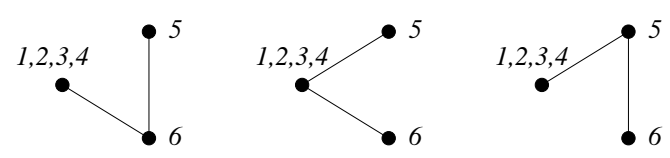

(d)

Figure $3 . G_{3}, G_{3} / C$ and their spanning trees

where $c$ is the number of cycles of $G$, and $\left\{i_{1}, i_{2}, \ldots, i_{j}\right\}$ are the sets of labels of cycles such that there is no $e_{x_{C a}}$ that lies on $C_{b}$ where $a \neq b$ and $a, b \in\left\{i_{1}, i_{2}, \ldots, i_{j}\right\}$.

In the formula above, we need to find the number of spanning forests of a graph. The Matrix tree theorem will help us to find the number of spanning trees of a connected graph, and we can use it to find the number of spanning forests.

Definition 4.10. Given an undirected graph $G$ with the vertices set $[n]=\{1,2, \ldots, n\}$, the Laplacian matrix $L(G)$ is defined to be $n \times n$ matrix with the following entries:

$$
l_{i, j}= \begin{cases}\operatorname{deg}(i) & \text { if } i=j, \\ -m & \text { where } m \text { is the number of edges between } i \text { and } j, \\ 0 & \text { otherwise. }\end{cases}
$$

When counting the degree of a vertex, all loops are excluded for multigraph.

Theorem 4.11 (The Matrix Tree Theorem, [8]). For a given undirected connected graph $G$ with $n$ vertices, let $\lambda_{1}, \ldots, \lambda_{n-1}$ be the nonzero eigenvalues of $L(G)$. Then the number of distinct spanning trees of $G$ is equal to

$$
t(G)=\frac{1}{n} \prod_{i=1}^{n-1} \lambda_{i}
$$


If $G$ is not connected with connected components $G_{1}, G_{2}, \ldots, G_{d}$, then it is not difficult to see that

$$
\left|\mathcal{B}_{G}\right|=\prod_{i=1}^{d} t\left(G_{i}\right) .
$$

Example 4.12. We will use Theorem 4.9 to find the number of decreasing maximal chains in $P_{G_{3}}$. We have

$$
L\left(G_{3}\right)=\left(\begin{array}{cccccc}
2 & -1 & -1 & 0 & 0 & 0 \\
-1 & 2 & 0 & -1 & 0 & 0 \\
-1 & 0 & 3 & -1 & -1 & 0 \\
0 & -1 & -1 & 3 & 0 & -1 \\
0 & 0 & -1 & 0 & 2 & -1 \\
0 & 0 & 0 & -1 & -1 & 2
\end{array}\right)
$$

The 5 non-zero eigenvalues of $L\left(G_{3}\right)$ are $1,2,3,3,5$. So the number of spanning trees of $G_{3}$ is $\frac{1 \cdot 2 \cdot 3 \cdot 3 \cdot 5}{6}=15$. Let $C_{1}$ be the cycle with vertices $1,2,3,4$, and $C_{2}$ be the cycle with vertices $2,3,5,6$, and $C_{3}$ be the cycle with vertices $1,2,5,6,3,4$. Since $G_{3} / C_{1}$ and $G_{3} / C_{3}$ are triangles, they both have 3 spanning trees. Since $G_{3} / C_{3}$ is a loop, it has 1 spanning tree. Since $e_{4}$ lies on $C_{1},\left|\mathcal{B}_{G / C_{1}, C_{2}}\right|=0$. Similarly, $\left|\mathcal{B}_{G / C_{1}, C_{3}}\right|=\left|\mathcal{B}_{G / C_{1}, C_{2}, C_{3}}\right|=0$. It is obvious that $\left|\mathcal{B}_{G / C_{2}, C_{3}}\right|=1$. Theorem 4.9 shows that the number of decreasing maximal chains in $P_{G_{3}}$ is

$$
15-(3+3+1-1-0-0+0)=9 .
$$

Now we provide several enumerative results for some classes of graphs.

Proposition 4.13. Let $G$ be a graph such that cycles in $G$ do not share edges. Then the number of decreasing maximal chains in $P_{G}$ is

$$
\prod_{k}(k-1)^{\alpha_{k}}
$$

where $\alpha_{k}$ is the number of $k$-cycles, $k \geq 3$.

Proof. Let $G^{\prime}$ be a spanning forest of $G$ that satisfies the conditions of Lemma 4.3. Then $G^{\prime}$ must contain $e_{x_{C}}$ for all cycles of $G$. Since $G^{\prime}$ is a spanning forest, it doesn't contain only one edge in $C \backslash e_{x_{C}}$ for every $C$. If $C$ is a cycle of length $k$, then we have $k-1$ choices for that edge. Therefore, the number of spanning forests satisfying the conditions of Lemma 4.3 is

$$
\prod_{k}(k-1)^{\alpha_{k}}
$$




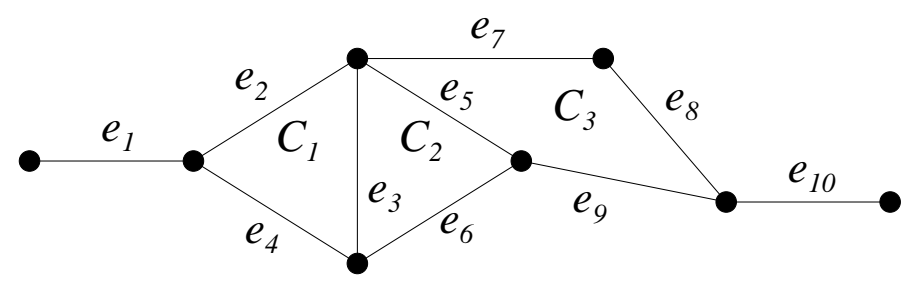

Figure 4. An example of $G$

Definition 4.14. A cycle $C$ is called a minimal cycle if $C$ doesn't contain the whole vertices of another cycle.

Proposition 4.15. Let $G$ be a graph such that all minimal cycles in $G$ can form a chain $C_{1}, C_{2}, \ldots, C_{s}$ where

$$
C_{i} \cap C_{j}= \begin{cases}\emptyset, \text { a vertex, or an edge } & \text { if } j=i+1, \\ \emptyset & \text { if } j \neq i+1 .\end{cases}
$$

Then the number of decreasing maximal chains in $P_{G}$ is

$$
\prod_{k}(k-1)^{\alpha_{k}}
$$

where $\alpha_{k}$ is the number of minimal cycles of length $k$.

Proof. Let $G$ be a graph satisfying the conditions above. Since $G$ does not contain a subdivision of $K_{5}$ or $K_{3,3}, G$ is a planar graph. Thus the chain of minimal cycles can be labeled from left to right as Figure 4.

We label the edges of $G$ in increasing order from the left to the right. If we meet a cycle, we label all edges of that cycle first before we move to other edges. This rule guarantees that every common edge of consecutive minimal cycles is the smallest edge of latter cycle.

Let $G^{\prime}$ be a spanning forest that satisfies the condition of Lemma 4.3. If an edge $e$ doesn't belong to any cycle, then $G^{\prime}$ must contain $e$.

Let $C$ be a minimal cycle of $G$ of length $k$. Since $G^{\prime}$ is a spanning forest, $G^{\prime}$ can contain at most $k-1$ edges of $C$. Since $G^{\prime}$ doesn't contain $C \backslash\left\{e_{x_{C}}\right\}$, there are $k-1$ ways to choose $(k-1)$-subsets of edges in $C$ in which we can choose some edges to put into $G^{\prime}$. Those subsets contain the smallest edge of $C$.

We construct a multiset $M$ that will contain edges of $G$ as follows. Firstly, we add all edges that are not in any cycle into $M$. In the next step, we choose a $(k-1)$-subset for every minimal cycle $C$ as described 
above to add into $M$. By the labeling rule of edges, every common edge of consecutive cycles must appear in $M$ at least once. Furthermore, each common edge can appear in $M$ at most twice because each edge can be a common edge of at most two cycles.

Now we prove that if we remove every common edge one time from the multiset $M$, we will get a set of edges that can form a spanning forest of $G$ satisfying the condition of Lemma 4.3. And also, whenever we change the subset of edges of a cycle, we will get another spanning forest.

By the construction, $M$ can not contain $C \backslash\left\{e_{x_{C}}\right\}$ for all $C$. Consider a maximal chain of minimal cycles $C_{j}, C_{j+1}, \ldots, C_{j+t}$ of $G$ such that $C_{j+i}$ and $C_{j+(i+1)}$ have a common edge or a common vertex for all $0 \leq i \leq t-1$. By the rule of labeling edges, each common edge of the consecutive cycles are the smallest edges of the latter cycle and it will appear in $M$. If $C_{j+i}$ has length $k_{j+i}, M$ contains a subset of $k_{j+i}-1$ edges of $C_{j+i}$. Let $\cup_{i} C_{j+i}$ be the union of $C_{j+i}$ for $0 \leq i \leq t$. If there are $x$ common vertices and $y$ common edges of minimal cycles in $\cup_{i} C_{j+i}$, then $\cup_{i} C_{j+i}$ has $\left(\sum_{i} k_{j+i}\right)-x-2 y$ vertices. The subsets of edges of $C_{j+i}$ form a multiset $N$ with $\left(\sum_{i} k_{j+i}\right)-t$ elements including edges of $\cup_{i} C_{j+i}$. When we remove the common edges of consecutive cycles in $N$ one time, we get a set $N^{\prime}$ with $\left(\sum_{i} k_{j+i}\right)-t-y$ edges. Since $t=x+y+1$, the set $N^{\prime}$ has $\left(\sum_{i} k_{j+i}\right)-x-2 y-1$ edges. Those edges form a subgraph of $\cup_{i} C_{j+i}$ including $\left(\sum_{i} k_{j+i}\right)-x-2 y$ vertices and $\left(\sum_{i} k_{j+i}\right)-x-2 y-1$ edges with no isolated vertex. Thus $N^{\prime}$ form a spanning tree of $\cup_{i} C_{j+i}$. Therefore we can conclude that when we remove every common edge one time in the multiset $M$, we will get a set of edges that can form a spanning forest of $G$ satisfying the condition of Lemma 4.3.

For each multiset $M$, the edges removed are the same, and they are all common edges in $G$. Since each edge except the common edges will appear in the subsets of edges of only one cycle, it will appear only once in $M$. Therefore, whenever we change the subset of edges of a minimal cycle, we will get another spanning forest.

On the other hand, let $T$ be a spanning tree of a connected component of $G$. We add common edges of cycles in that component into $T$ one time to get a multiset $N^{\prime \prime}$. Then we can partition $N^{\prime \prime}$ into a set of edges that are not in any cycle and $\left(k_{j}-1\right)$-subsets of cycles $C_{j}$ in the component.

Therefore, the number of spanning forests satisfying the condition of Lemma 4.3 is

$$
\prod_{k}(k-1)^{\alpha_{k}}
$$




\begin{tabular}{c|c} 
multisets $M$ & decreasing maximal chains \\
\hline$\{1,10,2,3,3,5,5,7,8\}$ & {$[10,8,7,5,3,2,1]$} \\
$\{1,10,2,3,3,5,5,7,9\}$ & {$[10,9,7,5,3,2,1]$} \\
$\{1,10,2,3,3,5,5,8,9\}$ & {$[10,9,8,5,3,2,1]$} \\
$\{1,10,2,3,3,6,5,7,8\}$ & {$[10,8,7,6,3,2,1]$} \\
$\{1,10,2,3,3,6,5,7,9\}$ & {$[10,9,7,6,3,2,1]$} \\
$\{1,10,2,3,3,6,5,8,9\}$ & {$[10,9,8,6,3,2,1]$} \\
$\{1,10,2,4,3,5,5,7,8\}$ & {$[10,8,7,5,4,2,1]$} \\
$\{1,10,2,4,3,5,5,7,9\}$ & {$[10,9,7,5,4,2,1]$} \\
$\{1,10,2,4,3,5,5,8,9\}$ & {$[10,9,8,5,4,2,1]$} \\
$\{1,10,2,4,3,6,5,7,8\}$ & {$[10,8,7,6,4,2,1]$} \\
$\{1,10,2,4,3,6,5,7,9\}$ & {$[10,9,7,6,4,2,1]$} \\
$\{1,10,2,4,3,6,5,8,9\}$ & {$[10,9,8,6,4,2,1]$}
\end{tabular}

TABLE 1. Multisets and decreasing maximal chains

where $\alpha_{k}$ is the number of minimal cycles of length $k$.

Example 4.16. Let $G$ be the graph with edge labeled shown in Figure 4. The subsets of edges of $C_{1}$ are $\{2,3\}$ and $\{2,4\}$. The subsets of edges of $C_{2}$ are $\{3,5\}$ and $\{3,6\}$. The subset of edges of $C_{3}$ are $\{5,8,9\},\{5,7,9\}$ and $\{5,7,8\}$. The common edges are 3,5 . Table 1 shows multisets and corresponding decreasing maximal chains. Thus there are 12 different decreasing maximal chains.

Proposition 4.17. Let $G$ be a graph such that we can label minimal cycles in $G$ to form a chain of cycles $C_{1}, C_{2}, \ldots, C_{s}$ of length $k_{1}, k_{2}, \ldots, k_{s}$ respectively. If

$$
C_{i} \cap C_{j}= \begin{cases}\emptyset, \text { a vertex, or an edge } & \text { if } i \neq t \text { and } j=i+1, \\ \alpha \text { edges }(\alpha>1) & \text { if } i=t \text { and } j=t+1, \\ \emptyset & \text { if } j \neq i+1,\end{cases}
$$

then the number of decreasing maximal chains in $P_{G}$ is

$$
\left[\left(k_{t}-1\right)\left(k_{t+1}-1\right)-\alpha(\alpha-1)\right] \prod_{i \neq t, t+1}\left(k_{i}-1\right) .
$$

Proof. Since $G$ is a planar graph, we can label cycles and edges in $G$ as in the proof of Proposition 4.15. Similar to Proposition 4.15, we add each $\left(k_{i}-1\right)$-subset of each $C_{i}$ into a multiset $M$, and remove common edges one time.

If a connected component that doesn't contain $C_{t}$ and $C_{t+1}$, the number of spanning trees is counted by Proposition 4.15. 
Consider the connected component that contains $C_{t}$ and $C_{t+1}$. Assume that this connected component of $G$ contains cycles

$$
C_{t-v}, C_{t-v+1}, \ldots, C_{t-1}, C_{t}, C_{t+1}, C_{t+2}, \ldots, C_{t+u} .
$$

Consider the case when $C_{t-1} \cap C_{t}, C_{t+1} \cap C_{t+2} \in\{\emptyset$, a vertex $\}$. By the labeling of edges, the smallest edge of $C_{t+1}$ is a common edge of $C_{t}$ and $C_{t+1}$. Since edges are labeled from the left to the right, the smallest edge of $C_{t}$ can not be a common edge of the two cycles. There are $k_{t}-\alpha$ and $k_{t+1}-\alpha$ edges of $C_{t}$ and $C_{t+1}$ respectively that are not common edges of $C_{t}, C_{t+1}$. If $\left(k_{t}-1\right)$-subset of $C_{t}$ and $\left(k_{t+1}-1\right)$-subset of $C_{t+1}$ added into $M$ contain all those edges, then after removing common edges we get a cycle. There are $\alpha$ ways to choose $\left(k_{t}-1\right)$-subsets of $C_{t}$ that contain all non-common edges of $C_{t}, C_{t+1}$. Since the smallest edge of $C_{t+1}$ is a common edge, there are $\alpha-1$ ways to choose $\left(k_{t+1}-1\right)$-subsets of $C_{t+1}$ that contain all non-common edges of the two cycles. So we have $\alpha(\alpha-1)$ ways of combination of subsets of $C_{t}$ and $C_{t+1}$ in which we have a cycle. Then the number of spanning trees of this component is

$$
\left[\left(k_{t}-1\right)\left(k_{t+1}-1\right)-\alpha(\alpha-1)\right] k_{t-v} \cdots k_{t-1} k_{t+2} \cdots k_{t+u} .
$$

The proof is similar to the proof of Proposition 4.15.

When $C_{t-1} \cap C_{t}=\{$ an edge $\}, C_{t+1} \cap C_{t+2} \in\{\emptyset$, a vertex $\}$, the common edge of $C_{t-1}$ and $C_{t}$ must be the smallest edge of $C_{t}$. If $M$ contains the $\left(k_{t-1}-1\right)$-subset of $C_{t-1}$ that does not contain the common edge of $C_{t-1}$ and $C_{t}$, and $\left(k_{t}-1\right)$-subset, $\left(k_{t+1}-1\right)$-subset of $C_{t}$ and $C_{t+1}$ respectively such that they contain all non-common edges of $C_{t}, C_{t+1}$, then we have a cycle. We also have $\alpha(\alpha-1)$ ways of combination of subsets of $C_{t}$ and $C_{t+1}$ as the above situation. Therefore, the number of spanning trees is

$$
\left[\left(k_{t}-1\right)\left(k_{t+1}-1\right)-\alpha(\alpha-1)\right] k_{t-v} \cdots k_{t-1} k_{t+2} \cdots k_{t+u} .
$$

When $C_{t-1} \cap C_{t} \in\{\emptyset$, a vertex $\}$ and $C_{t+1} \cap C_{t+2}=\{$ an edge $\}$, that edge must be the smallest edge of $C_{t+2}$. Every $\left(k_{t+2}-1\right)$-subset of $C_{t+2}$ contains that common edge. Similarly, we also have

$$
\left[\left(k_{t}-1\right)\left(k_{t+1}-1\right)-\alpha(\alpha-1)\right] k_{t-v} \cdots k_{t-1} k_{t+2} \cdots k_{t+u}
$$

spanning trees.

When $C_{t+1} \cap C_{t+2}=$ an edge $\}$ and $C_{t-1} \cap C_{t}=$ an edge $\}$, the common edge of $C_{t+1}$ and $C_{t+2}$ must be the smallest edge of $C_{t+2}$. If $M$ contains the $\left(k_{t-1}-1\right)$-subset of $C_{t-1}$ that does not contain the common edge of $C_{t-1}$ and $C_{t}$, and $\left(k_{t}-1\right)$-subset, $\left(k_{t+1}-1\right)$-subset of $C_{t}$ and $C_{t+1}$ respectively such that they contain all non-common edges of $C_{t}, C_{t+1}$, 
then after removing the common edges we also have a cycle. So the number of spanning trees of this component is

$$
\left[\left(k_{t}-1\right)\left(k_{t+1}-1\right)-\alpha(\alpha-1)\right] k_{t-v} \cdots k_{t-1} k_{t+2} \cdots k_{t+u} .
$$

Consequently, the number of spanning forests that satisfy the condition of Lemma 4.3 is

$$
\left[\left(k_{t}-1\right)\left(k_{t+1}-1\right)-\alpha(\alpha-1)\right] \prod_{k_{i} i \neq t, t+1}\left(k_{i}-1\right) .
$$

Proposition 4.18. Let $G$ be a graph such that in each connected component all minimal cycles share only one common edge. Then the number of decreasing maximal chains of the intersection poset of graphical arrangement of $G$ is equal to

$$
\prod_{k}(k-1)^{\alpha_{k}}
$$

where $\alpha_{k}$ is the number of minimal cycles of length $k$.

Proof. In each connected component, we label edges so that the common edge is the smallest edge of all minimal cycles in that component. We construct a multi set $M$ by adding all edges that do not belong to any cycle and adding one $(k-1)$-subset of each minimal cycle $C$ of length $k$. If $e$ is a common edge of $x$ cycles, then $e$ will appear $x$ times in $M$. We remove $e x-1$ times to get a set $N$ of edges in $G$. We prove that edges in $N$ form a spanning forest that satisfies the condition of Lemma 4.3.

It is obvious that $N$ doesn't contain $C \backslash\left\{e_{x_{C}}\right\}$ for all $C$.

Let $C_{1}, C_{2}, \ldots, C_{t}$ be all minimal cycles of a connected component. Let $\cup_{i} C_{i}$ be the union of $C_{1}, C_{2}, \ldots, C_{t}$. Assume that $C_{1}, C_{2}, \ldots, C_{t}$ have length $k_{1}, k_{2}, \ldots, k_{t}$ respectively. Then the number of vertices in $\cup_{i} C_{i}$ is $\left[\sum_{i=1}^{t} k_{i}\right]-2(t-1)$. After removing edges, the number of edges of $\cup C_{i}$ in $N$ is $\left(\sum_{i=1}^{t}\left(k_{i}-1\right)\right)-(t-1)=\left(\sum_{i=1}^{t} k_{i}\right)-2 t+1$. Since the subgraph formed by edges in $N$ doesn't contain isolated vertices, the edges in $N$ form a spanning tree of $\cup_{i} C_{i}$.

Whenever we change a $\left(k_{i}-1\right)$-subset of $C_{i}$ in $M$, we get a new spanning tree of $\cup_{i} C_{i}$.

On the other hand, let $T$ be a spanning tree of a connected component of $G$ which has $t$ cycles. We add the common edge of those cycles $t-1$ times into $T$ to get a multiset $N^{\prime}$. Then we can partition $N^{\prime}$ into a set 
of edges that are not in any cycle and $\left(k_{i}-1\right)$-subsets of cycles $C_{i}$ in the component.

Therefore, the number of spanning forests of $G$ satisfying condition of Lemma 4.3 is

$$
\prod_{k}(k-1)^{\alpha_{k}}
$$

where $\alpha_{k}$ is the number of minimal cycles of length $k$.

\section{The number of regions}

An application of finding the number of decreasing maximal chains is counting regions of an arrangement. Recall that a region of an arrangement $\mathcal{A}$ is a connected component of the complement $X$ of the hyperplanes,

$$
X=\mathbb{R}^{n}-\cup_{H \in \mathcal{A}} H .
$$

By Zaslavsky, the number of regions of an arrangement is found by the formula.

Theorem 5.1 (see [10]). Let $\mathcal{A}$ be a hyperplane arrangement in $\mathbb{R}^{n}$. Then

$$
\#\{\text { regions }\}=\sum_{x \in P}|\mu(\hat{0}, x)|,
$$

where $P$ is the intersection poset of arrangement $\mathcal{A}$ and $\mu$ is the Möbius function of $P$.

The following theorem describes the number of regions of graphical arrangement.

Theorem 5.2. The number of regions of the graphical arrangement of a graph $G$ is equal to

$$
\sum_{H} d_{H}
$$

where the sum is over all subgraphs $H$ of $G$ whose connected components are induced subgraphs with at least two vertices and $d_{H}$ is the number of decreasing maximal chains in the intersection poset $P_{H}$.

Proof. Let $A=A_{1}-A_{2}-\cdots-A_{p}$ be an element of the intersection poset $P_{G}$ where $A_{i}, i=1,2, \ldots, p$, are blocks of $A$. Each $A_{i}$ contains indices or vertices of $G$. Let $G_{i}$ be the subgraph induced by $A_{i}$. Let $G_{A}$ be the union of subgraphs $G_{i}, i=1,2, \ldots, p$. Then for each element $A$ of $P_{G}$, we get a subgraph $G_{A}$ of $G$. It is obvious that the intersection poset $P_{G_{A}}$ is isomorphic to the interval $[\hat{0}, A]$ of $P_{G}$ (see [5, Lemma 2.11]). By 
[9, Theorem 4.11], $|\mu(\hat{0}, A)|$ is equal to the number $d_{G_{A}}$ of decreasing maximal chains of $P_{G_{A}}$. By Theorem 5.1, the number of regions of $\mathcal{A}_{G}$ is

$$
\sum_{A \in P_{G}}|\mu(\hat{0}, A)|=\sum_{A \in P_{G}} d_{G_{A}} .
$$

Since each block of an element $\mathcal{A}$ in $P_{G}$ can be associated with an induced subgraph of $G$ with at least two vertices, the result follows.

Now we provide some enumerative results.

Proposition 5.3. The number of regions of graphical arrangement of a forest with $m$ edges is $2^{m}$

Proof. It is easy to see that the intersection poset of $\mathcal{A}_{G}$, where $G$ is a forest, has only one decreasing maximal chain, and the intersection poset of arrangement of every its subgraph also has one decreasing maximal chain. To find the number of regions, we need to find the number of subgraphs of $G$ satisfying the condition of Theorem 5.2. Since the union of any edges can give a subgraph satisfying the condition of Theorem 5.2, the number of regions is the number of subsets of edges, and equal to $2^{m}$.

Remark 5.4. In general, a graphical arrangement is not in general position, but the graphical arrangement for a forest is in general position. It is known that if an arrangement of $m$ hyperplanes in $\mathbb{R}^{n}$ is in general position, then the number of regions is found by the formula (see [9, Proposition 2.4])

$$
1+m+\left(\begin{array}{c}
m \\
2
\end{array}\right)+\cdots+\left(\begin{array}{c}
m \\
n
\end{array}\right) .
$$

So we can also use this formula to show Proposition 5.3.

Proposition 5.5. Let $G$ be a graph with $m$ edges and a unique cycle $C$ of length $k$. Then the number of regions of graphical arrangement of $G$ is $2^{m}-2^{m-k+1}$.

Proof. We would like to count the number of subgraphs of $G$ satisfying the conditions of Theorem 5.2.

First, consider the case when the subgraph $H$ does not contain $C$. If $H$ contains all but one edge of $C$, the connected component of $H$ containing those edges is not an induced subgraph. Since there are $2^{m-k}$ subgraphs of $G$ containing $C$ and $k 2^{m-k}$ subgraphs of $G$ containing all but one edge of $C$, there are $2^{m}-(k+1) 2^{m-k}$ subgraphs of $G$ with no cycle which satisfy the condition of Theorem 5.2. 
Since any subgraph of $G$ containing $C$ satisfies the condition of Theorem 5.2, there are $2^{m-k}$ subgraphs of $G$ containing $C$.

By Proposition 4.13, the intersection poset of graphical arrangement of graph with no cycle has 1 decreasing maximal chain, and the number of decreasing maximal chains is $k-1$ when the graph has one cycle of length $k$. Theorem 5.2 implies the number of regions is

$$
2^{m}-(k+1) 2^{m-k}+(k-1) 2^{m-k}=2^{m}-2^{m-k+1} .
$$

Proposition 5.6. Let $G$ be a graph with $m$ edges and $s$ cycles $C_{1}, C_{2}, \ldots, C_{s}$ of length $k_{1}, k_{2}, \ldots, k_{s}$ respectively such that they do not share edges. Then the number of regions of graphical arrangement of $G$ is

where

$$
\sum_{Y \subseteq[s]}\left[S_{Y} \prod_{j \in Y}\left(k_{j}-1\right)\right]
$$

$$
S_{Y}=2^{m-\sum_{j \in Y} k_{j}}-\sum_{\emptyset \neq X \subseteq[s] \backslash Y}\left[\left(\prod_{j \in X} k_{j}+1\right) 2^{m-\sum_{j \in Y} k_{j}-\sum_{j \in X} k_{j}}\right]
$$

for subsets $X, Y$ of $[s]$.

Proof. We would like to count the number of subgraphs of $G$ satisfying the conditions of Theorem 5.2.

Consider the case when the subgraph $H$ does not contain any cycle. If $H$ contains all but one edge of any cycle, the connected component of $H$ containing those edges is not an induced subgraph. For $r$ cycles $C_{x_{1}}, C_{x_{2}}, \ldots, C_{x_{r}}$, there are $2^{m-k_{x_{1}}-\cdots-k_{x_{r}}}$ subgraphs containing $C_{x_{1}}, C_{x_{2}}, \ldots, C_{x_{r}}$ and $k_{x_{1}} k_{x_{2}} \cdots k_{x_{r}} 2^{m-k_{x_{1}}-\cdots-k_{x_{r}}}$ subgraphs containing all but one edge in each cycle $C_{x_{1}}, C_{x_{2}}, \ldots, C_{x_{r}}$. Thus the number of subgraphs of $G$ with no cycle which satisfy the condition of Theorem 5.2 is

$$
\begin{aligned}
S & =2^{m}-\sum_{r=1}^{s}\left[\sum_{x_{1}, x_{2}, \ldots, x_{r}}\left[\left(\prod_{q=1}^{r} k_{x_{q}}+1\right) 2^{m-\sum_{q=1}^{r} k_{x_{q}}}\right]\right. \\
& =2^{m}-\sum_{\emptyset \neq X \subseteq[s]}\left[\left(\prod_{j \in X} k_{j}+1\right) 2^{m-\sum_{j \in X} k_{j}}\right]
\end{aligned}
$$

When $H$ contains cycles $C_{y_{1}}, C_{y_{2}}, \ldots, C_{y_{u}}$ for $y_{1}, y_{2}, \ldots, y_{u} \in Y \subseteq[s]$, we have

$$
S_{Y}=2^{m-\sum_{j \in Y} k_{j}}-\sum_{\emptyset \neq X \subseteq[s] \backslash Y}\left[\left(\prod_{j \in X} k_{j}+1\right) 2^{m-\sum_{j \in Y} k_{j}-\sum_{j \in X} k_{j}}\right]
$$


subgraphs of $G$ containing $C_{y_{1}}, C_{y_{2}}, \ldots, C_{y_{u}}$ which satisfy the condition of Theorem 5.2.

By Proposition 4.15 and Theorem 5.2, the number of regions is

$$
S+\sum_{\emptyset \neq Y \subseteq[s]}\left[S_{Y} \prod_{j \in Y}\left(k_{j}-1\right)\right] .
$$

Since $S=S_{Y}$ when $Y=\emptyset$, the result follows.

\section{References}

[1] Anders Björner, Shellable and Cohen-Macaulay partially ordered sets, Trans. Amer. Math. Soc., 260(1) (1980), 159-183.

[2] Anders Björner and Michelle L. Wachs, Shellable nonpure complexes and posets. I, Trans. Amer. Math. Soc., 348(4) (1996), 1299-1327.

[3] Curtis Greene and Thomas Zaslavsky, On the interpretation of Whitney numbers through arrangements of hyperplanes, zonotopes, non-Radon partitions, and orientations of graphs, Trans. Amer. Math. Soc., 280(1) (1983), 97-126.

[4] Peter Orlik and Louis Solomon, Combinatorics and topology of complements of hyperplanes, Invent. Math., 56(2) (1980), 167-189.

[5] Peter Orlik and Hiroaki Terao, Arrangements of hyperplanes, volume 300 of Grundlehren der Mathematischen Wissenschaften [Fundamental Principles of Mathematical Sciences], Springer-Verlag, Berlin, 1992.

[6] Richard P. Stanley, Supersolvable lattices, Algebra Universalis, 2 (1972), 197-217.

[7] Richard P. Stanley, Finite lattices and Jordan-Hölder sets, Alg. Univ., 4 (1974), 361-371.

[8] Richard P. Stanley, Enumerative Combinatorics, vol 2, Cambridge Studies in Advanced Mathematics, 62, Cambridge University Press, 1999.

[9] Richard P. Stanley, An introduction to hyperplane arrangements, In Geometric combinatorics, volume 13 of IAS/Park City Math. Ser., pages 389-496. Amer. Math. Soc., Providence, RI, 2007.

[10] Thomas Zaslavsky, Facing up to arrangements: face-count formulas for partitions of space by hyperplanes, Mem. Amer. Math. Soc., 1(issue 1, 154):vii+102, 1975.

[11] Michelle L. Wachs, A basis for the homology of the d-divisible partition lattice, Adv. Math., 117(2) (1996), 294-318.

[12] Michelle L. Wachs, Poset topology: tools and applications, In Geometric combinatorics, volume 13 of IAS/Park City Math. Ser., pages 497-615. Amer. Math. Soc., Providence, RI, 2007.

Thi A. Nguyen

Department of Mathematics, Chonnam National University, Gwangju 500-757, Republic of Korea.

E-mail: thinguyen16@gmail.com 
Sangwook Kim

Department of Mathematics, Chonnam National University, Gwangju 500-757, Republic of Korea.

E-mail: swkim.math@chonnam.ac.kr 